\title{
Random Unitary Matrices Associated to a Graph
}

\author{
P. KONDRATIUK ${ }^{a, *, \dagger}$ AND K. ŻYCZKOWSKI ${ }^{b, c}$ \\ ${ }^{a}$ Faculty of Physics, Warsaw University of Technology, Koszykowa 75, 00-662 Warsaw, Poland \\ ${ }^{b}$ Institute of Physics, Jagiellonian University, W.S. Reymonta 4, 30-059 Kraków, Poland \\ ${ }^{c}$ Center for Theoretical Physics, PAS, al. Lotników 32/44, 02-668 Warszawa, Poland
}

\begin{abstract}
We analyze composed quantum systems consisting of $k$ subsystems, each described by states in the $n$-dimensional Hilbert space. Interaction between subsystems can be represented by a graph, with vertices corresponding to individual subsystems and edges denoting a generic interaction, modeled by random unitary matrices of order $n^{2}$. The global evolution operator is represented by a unitary matrix of size $N=n^{k}$. We investigate statistical properties of such matrices and show that they display spectral properties characteristic to the Haar random unitary matrices provided the corresponding graph is connected. Thus basing on random unitary matrices of a small size $n^{2}$ one can construct a fair approximation of large random unitary matrices of size $n^{k}$. Graph-structured random unitary matrices investigated here allow one to define the corresponding structured ensembles of random pure states.
\end{abstract}

DOI: $10.12693 /$ APhysPolA.124.1098

PACS: 02.10.Yn, 03.65.Aa, 03.67.Mn, 05.45.Mt, 05.45.Pq

\section{Introduction}

Random unitary matrices can be applied to describe quantum chaotic scattering or an evolution operator for a periodic, time-dependent system, the corresponding classical dynamics is chaotic in the entire phase space [1]. If the system possesses no time-reversal symmetry, the corresponding operators display statistical properties typical to circular unitary ensemble (CUE) of matrices distributed according to the Haar measure on the unitary group [2].

A random matrix typical to CUE is hence related to a common physical situation, in which there exist a generic, possibly unspecified, interaction between any two levels of the entire system. In a more general setup of a multi-partite system this assumption corresponds thus to a typical interaction between any pair of subsystems.

On the other hand, in a broad class of quantum models studied in condensed matter or atomic physics the interaction acts only locally between neighbouring particles on a prescribed lattice. If the exact Hamiltonian describing such an interaction is unknown, one can mimic it by a random unitary matrix which couples only a few selected subsystems. In this way we arrive at a model of random unitary matrices associated to a graph or a network, which will be introduced and analyzed in this work.

The model described precisely in the next section, is related to the ensemble of structured quantum pure states associated with a graph investigated in $[3,4]$. These assumptions differ significantly from the model analyzed

*corresponding author; e-mail: Pawel.Kondratiuk@fuw.edu.pl

†Present address: Institute of Theoretical Physics, Faculty of Physics, University of Warsaw, Hoża 69, 00-681 Warsaw, Poland. in [5], in which edges of the graph represent maximally entangled states of two qubits, while the vertices represent deterministic local unitary gates or local measurements. A similar idea of an edge representing a maximally entangled state of two particles was also used in a deterministic construction of projected entangled pair states [6], while more general models of quantum networks were analyzed in $[7,8]$.

We extend here the model introduced in [3] of a random unitary interaction represented by each vertex of the graph, but make the model symmetric, by assuming that in the subsequent time step a similar random interaction takes place along each bond of the graph. Thus the physical role of bonds and edges of a graph is in sense similar, in an analogy to the construction of line-graphs [9].

The main aim of this work is to introduce ensembles of structured random unitary matrices associated to a graph and to investigate their basic properties. We report here a key observation concerning the spectral statistics of such structured unitary matrices. On the one hand, ensembles of matrices related to non-connected graphs display Poisson-like spectra. On the other hand, a typical connected graph leads to an ensemble with several properties characteristic to CUE, even though the interaction takes place locally between the subsystems connected by a bond or belonging to a single vertex of a graph.

The paper is organised as follows. In the next section two alternative versions of the scheme associating a random unitary matrix to a graph are described. Statistical properties of spectra of random matrices corresponding to exemplary graphs are analyzed in Sect. 3. The distribution of eigenvectors of graph unitary matrices is analyzed in Sect. 4, while statistical properties of their entries are discussed in Sect. 5. Concluding remarks are presented in Sect. 6, while some details concerning the numerical computations are provided in Appendix. 


\section{Interactions associated to a graph and corresponding unitary matrices}

We are going to discuss a general case of a composite quantum system consisting of an arbitrary number $k$ of subsystems isolated from the environment. For simplicity we shall assume here that each subsystem is described in an $n$-dimensional Hilbert space, $\mathcal{H}_{n}$. Hence the total dimension of the Hilbert space reads $N=n^{k}$ and the composite system is described by a state $|\psi\rangle$ in the composite Hilbert space $\mathcal{H}_{N}=\mathcal{H}_{n} \otimes \cdots \otimes \mathcal{H}_{n}$.

A Hamiltonian evolution operator can be represented by a global unitary matrix $U$ of order $N$. Assume first that the time evolution of the composite system can be decomposed into two time steps, so the time evolution is given by a product of two matrices

$$
U=W V \text {. }
$$

Here $W$ and $V$ denote unitary matrices, which describe both phases of the time evolution, which occurs sequentially, one after another.

The main assumption of the model is that the physical interactions taking place between certain subsystems has a topology which can be described by a graph. To make the presentation more complete we shall define two different schemes of representing the interaction by a graph. Although some interaction patterns can be described equally well using any one of the two constructions proposed, in some cases only one of these two schemes is applicable, which provides a motivation to describe both of them.

\subsection{A bond of a graph represents two coupled subsystems}

In the first approach we will assume that the total number of subsystems is even $k=2 m$ and the interaction can be represented by an undirected graph $\Gamma_{1}$ consisting of $m$ bonds and $v$ vertices. In general, the graph needs not to be connected and we may allow loops and multiple connections between vertices.

In the first time step of the evolution a generic interaction takes place independently in each vertex of the graph. Such an interaction is described by a random unitary operator $V^{(j)}$, where $j=1, \ldots, v$ labels the vertices of the graph. For instance, if the first vertex couples the subsystems labeled by 2 and 3 we shall write $V^{(1)}=V_{2,3}$. The interaction in all vertices is thus described by a tensor product

$$
V=V^{(1)} \otimes V^{(2)} \otimes \ldots \otimes V^{(v)} .
$$

Each bond of a graph represents two subsystems interacting jointly in the second time step. For instance, the first bond, connecting subsystems labeled by 1 and 2, will denote a generic interaction between them represented by a random unitary matrix $W_{12}$ of order $n^{2}$. Hence the second time step is described by a unitary matrix of the product form

$$
W=W_{1,2} \otimes W_{3,4} \otimes \ldots \otimes W_{k-1, k},
$$

where $W_{2 j-1,2 j}$ describes a generic bi-partite interaction corresponding to $j$-th bond of the graph. Hence the entire, two-step time evolution reads $U=W V$, according to (1), where both unitary terms $W$ and $V$ have a tensor product structure. Observe that the tensor product symbols $\otimes$ present in (2) are taken with respect to different partitions of the total Hilbert space as these occurring in Eq. (3), so in general the operator $U$ does not possess a tensor product structure.

To watch this construction in action consider a simple graph consisting of two vertices, $v=2$ and two bonds between them, $m=2$. This graph describes thus $k=2 m=4$ subsystems, which are labeled here by 1,2 , 3, 4 - see Fig. 1. The evolution operator constructed according to the rules $(1,2,3)$ reads thus

$$
U=\left(W_{1,2} \otimes W_{3,4}\right)\left(V_{2,3} \tilde{\otimes} V_{1,4}\right),
$$

where the interaction along the bonds is given by random unitary matrices $W_{1,2}$ and $W_{3,4}$ of size $n^{2}$, while interaction at the vertices is described by unitary matrices $V_{2,3}$ and $V_{1,4}$ of the same size. It is convenient to label unitary matrices $V$, describing interaction at a given vertex by its number, written in a superscript in brackets or by the numbers of particle it includes placed in a subscript, and freely switch between both conventions. In the example described above one has $V^{(1)}=V_{2,3}$ and $V^{(2)}=V_{1,4}$.
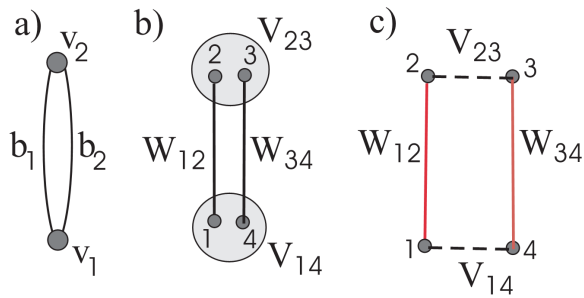

Fig. 1. (a) An exemplary graph with two vertices and two bonds describes a 4-party system, (b) construction of the corresponding random unitary matrix $U$ defined by Eq. (4); (c) the same interaction described by Eq. (5) and represented by a two-color graph - one color is represented by solid (red) lines and the other by dashed (black) lines.

The sign tilde over the second tensor product in Eq. (4) is put to emphasize that both tensor products are taken with respect to different partitions, so $U$ cannot be written as a tensor product of two local unitary matrices.

\subsection{A vertex of a two-color graph represents a subsystem}

In the second approach each of $v$ vertices of the graph describes a single subsystem, hence the number of parties involved $k=v$ is arbitrary. On the other hand, in this scheme we need to make some restrictions concerning the topology of the graph representing the interaction. To describe physical interaction occurring in two moments of time we will use two kinds of bonds, denoted in the graph by two different colours. This construction is unambiguous provided the two-colour graph $\Gamma_{2}$ considered here satisfies the following property: there exists 
a single bond of each colour linked to a given vertex (see Fig. 2b-e), or if there are more of them (for example two red, solid bonds entering vertex 1 in Fig. 2f), they are a part of a maximally connected (sub)graph of this colour (a triangle in this figure).
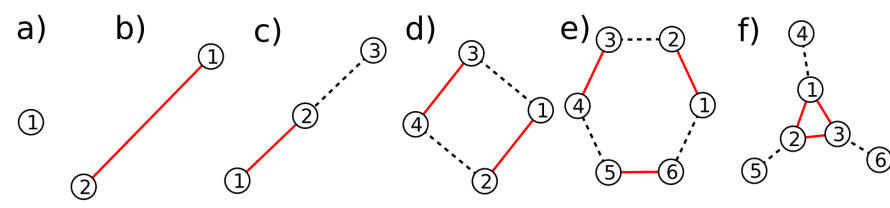

Fig. 2. Examples of two-colour interaction graphs, representing due to Eq. (5) different evolution operators: (a) $U=V_{1} \in \mathrm{U}(n),\left(\right.$ b) $U=W_{12}\left(V_{1} \otimes V_{2}\right) \in$ $\mathrm{U}\left(n^{2}\right)$, (c) $U=\left(W_{12} \otimes W_{3}\right)\left(V_{1} \otimes V_{23}\right) \in \mathrm{U}\left(n^{3}\right),(\mathrm{d})$ $U=\left(W_{12} \otimes W_{34}\right)\left(V_{13} \otimes V_{24}\right) \in \mathrm{U}\left(n^{4}\right), \quad(\mathrm{e}) U=$ $\left(W_{12} \otimes W_{34} \otimes W_{56}\right)\left(V_{23} \otimes V_{45} \otimes V_{61}\right) \in \mathrm{U}\left(n^{6}\right),(\mathrm{f})$ $U=\left(W_{123} \otimes W_{4} \otimes W_{5} \otimes W_{6}\right)\left(V_{14} \otimes V_{25} \otimes V_{36}\right) \in \mathrm{U}\left(n^{6}\right)$. Graphs (a) and (b) represent structureless (CUE) matrices, while graphs (c)-(f) correspond to structured matrices.

To present a formal definition of this property we will use notation of the graph theory. A clique in the graph is defined as a subset of vertices connected to each other. Let $Q=\left\{q_{i}\right\}_{i=1}^{k}$ denotes the set of vertices of the graph. A partition of the set $Q$, given by any set of its mutually exclusive and collectively exhaustive subsets is denoted by $\Pi(Q)$. Our requirement concerning the graph $\Gamma_{2}$ is then equivalent to an assumption that its vertices can be divided into two partitions, $\Pi_{1}(Q)$ and $\Pi_{2}(Q)$, both of which consist of separate cliques only. Each partition is represented on the graph by bonds of a certain color. Hence we introduce two sets of bonds, $B_{1}$ and $B_{2}$, and represent the evolution operator by two graphs of interactions, $G_{1}=\left\{Q, B_{1}\right\}$ and $G_{2}=\left\{Q, B_{2}\right\}$.

The unitary operator $U$ of the entire system, describing the two-step time evolution, can be therefore expressed as

$$
U=\left(\bigotimes_{\pi \in \Pi_{2}(Q)} W_{\pi}\right)\left(\tilde{\bigotimes}_{\pi \in \Pi_{1}(Q)} V_{\pi}\right)
$$

where operator $V_{\pi}$ (or $W_{\pi}$ ) acts on the particles from the subset $\pi$, and the tilde over the sign $\otimes$ in the second term is used to emphasize that the tensor products are taken with respect to different partitions. The size of a component unitary matrix $V_{\pi}$ is a function of the number $\# \pi$ of the particles in the subset $\pi$ as $\operatorname{dim} V_{\pi}=n^{\# \pi}$. Note that the operator (5) is now uniquely determined by a two-color graph $\Gamma_{2}=\left\{Q,\left\{B_{1}, B_{2}\right\}\right\}$ (consisting of a set of vertices and two sets of edges).

Some examples of the two-colour graphs satisfying the cliques assumption and representing evolution operators are shown in Fig. 2. The interactions are represented by either black dashed or red solid edges. Observe that the system represented by the graph Fig. $2 d$ was already described by the former construction and shown in Fig. 1. On the other hand, the former approach, in which each bond represents two subsystems, is not applicable for the system visualised in Fig. 2c which consists of an odd number of subsystems.

It is straightforward to generalize the above construction for three (or more) steps of the time evolution, which is determined by a graph consisting of three (or more) classes of bonds.

\section{Spectral properties of graph-structured matrices}

Eigenvalues of a unitary matrix of order $N$ lie on the unit circle in the complex plane, so they have the form $z_{j}=\mathrm{e}^{\mathrm{i} \theta_{j}}$ for $j=1, \ldots, N$. One can thus consider the probability density of the eigenphases, $P(\theta)$, which is known to be uniform in $[0,2 \pi)$ for random matrices of circular ensembles [2].

Diagonalisation of a unitary matrix $U$ of order $N$ yields $N$ eigenphases. After they are sorted, $\theta_{1} \leq \theta_{2} \leq \cdots \leq$ $\theta_{N}$, one may consider the normalized nearest neighbour spacing,

$$
S_{i}=\frac{N}{2 \pi}\left(\theta_{i+1}-\theta_{i}\right) .
$$

Each circular ensemble of random matrices (Poisson, orthogonal, unitary and symplectic) is characterized by the specific level spacing distribution $P(S)$. In the case of the unitary ensemble (CUE), equivalent to the Haar measure on the unitary group, one can use the Wigner surmise

$$
P(S)=\frac{32}{\pi^{2}} S^{2} \exp \left(-\frac{4}{\pi} S^{2}\right),
$$

which is exact for random unitary matrices of order 2 and gives a good approximation [2] also for large matrices, $N \rightarrow \infty$.

We were examining the spectral properties of various graph-structured matrices, built of smaller CUE matrices, which describe the interaction along the bonds or in the vertices. For simplicity we will focus our attention here on systems which can be represented by two-color graphs defined in Sect. 2.2, and will use the graphical convention described in that section. We constructed numerically ensembles of structured random unitary matrices corresponding to graphs presented in Fig. 3. Random unitary matrices [10] used as building blocks of the construction presented were obtained by the algorithm of Mezzadri [11] — some details concerning the numerical procedure are provided in Appendix. In all cases studied the level density $P(\theta)$ distribution is uniform, which is the case for standard ensembles of random unitary matrices.

Regarding the structure of the graph ${ }^{\ddagger}$, we distinguish two cases:

$¥$ When we refer to (dis) connectivity of a graph, we disregard the fact that we actually have two (or more) different sets of bonds $B_{i}$. So in fact we are interested in the problem of connectivity of the graph $\Gamma=\left(Q, \cup_{i} B_{i}\right)$, where all the sets of bonds corresponding to the different stages of interactions have been summed. 


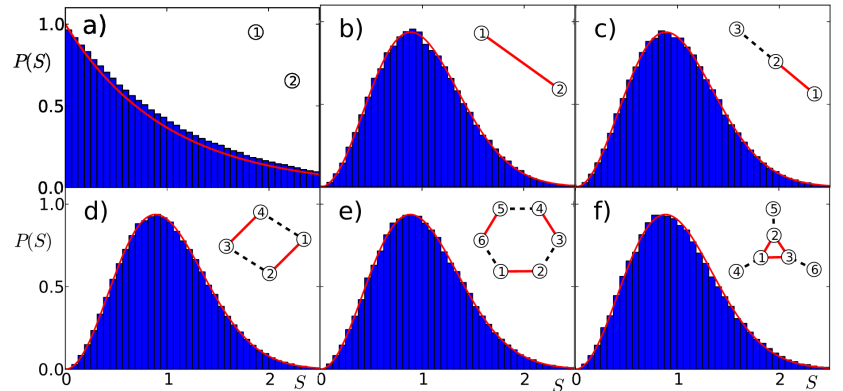

Fig. 3. Nearest level spacing statistics $P(S)$ for the unitary matrices corresponding to the graphs shown in the inset. For a disconnected graph (a) the matrices $U$ display Poissonian spectra, while for connected graphs (b)-(f) the level spacing distribution is described by the Wigner distribution (7) characteristic of CUE. The matrix size $N$ reads (a) $N=256$, (b) $N=100$, (c) $N=1000$, (d) $N=2401$, (e) $N=729$, (f) $N=64$. In each case, the number of generated eigenvalues was of the order of $10^{5}$.

- The graph is disconnected. In this case the matrix $U$ can be written as the Kronecker (tensor) product of two or more smaller matrices. The resulting level spacing distribution is similar to Poissonian ensemble, for which the eigenvalues are uncorrelated.

- The graph is connected. Our numerical results show that several properties of the structured unitary matrices corresponding to connected graphs are similar to those of random structureless matrices. In particular, if all the component matrices $W_{\pi}$ and $V_{\pi}$ are taken according to CUE, the structured evolution matrix $U$ defined in Eq. (5) displays spectral properties characteristic to the Haar measure on $U(N)$ with $N=n^{k}$. Figure 3 presents spacing distributions $P(S)$ obtained for the matrices determined by exemplary graphs shown in each figure.

It is possible to relate these observations with recent results on tensor products of random unitary matrices, which display Poissonian level spacing in the limit of large matrices [12-14]. Asymptotically both tensor product factors, $W=\otimes W_{\pi_{1}}$ and $V=\otimes V_{\pi_{2}}$ in Eq. (5) display thus a Poissonian spectra, so the evolution operator represented in the eigenbasis of the first term has the form $Y^{\dagger} U Y=P_{1} X P_{2} X^{\dagger}$. Here $P_{1}$ and $P_{2}$ denote diagonal unitary matrices with Poissonian spectra of $W$ and $V$, respectively. The unitary matrices $Y$ and $X$ are determined by eigenvectors of $W$ and $V$.

Note that unitary rotation matrix $Y$ does not influence the spectrum of $U$. In the case of a connected graph the tensor products defining $W$ and $V$ are taken with respect to different partitions, hence it is possible to assume that the rotation matrix $X$ constructed out of eigenvectors has a CUE like properties [1]. Hence we arrive at a composed ensemble of matrices [15], of the form $U^{\prime}=P_{1} X P_{2} X^{\dagger}$, which contains a product of two diagonal matrices repre- sented in different (random) bases. Although both matrices $P_{1}$ and $P_{2}$ possess Poissonian level spacing distributions, the composed ensemble displays CUE-like spectra, which explains the results obtained for matrices structured by connected graphs. We performed numerical investigation for unitary matrices of size $N=100$ of this composed ensemble and found that level spacing distribution of $U^{\prime}$ fits well to predictions of random matrices.

In the case of disconnected graph the both terms $W$ and $V$ in Eq. (5) have a tensor product structure with respect to the same partition, for instance $U=$ $\left(W \otimes W^{\prime}\right)\left(V \otimes V^{\prime}\right)=W V \otimes W^{\prime} V^{\prime}$. Thus the evolution operator $U$ has a tensor product form, so its level spacing distribution becomes asymptotically Poissonian [12], which explains properties of matrices structured by disconnected graphs.

The above arguments work asymptotically for evolution operators describing the interaction represented by two-colour connected graphs, such that each vertex contains at least one connection of each colour. To analyze to what extent this assumption can be relaxed we investigated an $L$ step interaction, described by a larger class of graphs with bonds of $L$ different colours. In the simplest case, consider a chain of $L+1$ subsystems, such that in each moment only two neighbouring subsystems are involved: in the first step the first subsystem interacts with the second, in the second step the interaction couples subsystems two and three, and so on.

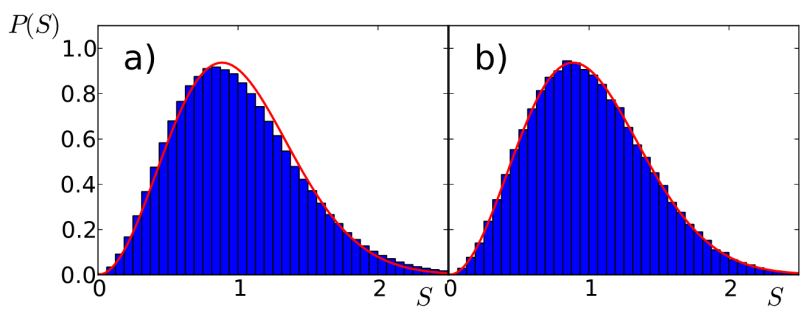

Fig. 4. Level spacing distribution $P(S)$ for unitary matrices $U$ corresponding to a five-step evolution of a six-chain consisting of (a) six qubits, $n=2$ and (b) six qutrits, $n=3$. The sample size is (a) 20000 and (b) 400 matrices. In the $i$-th step of the evolution only $i$-th and $(i+1)$-th subsystem interact so $U$ is given by Eq. (8).

This interaction can be represented by a chain of $L+1$ vertices, such that all $L$ bounds between the neighbouring vertices are of a different colour, associated with the interaction in specific time steps. Figure 4 shows the level spacing distribution for such a model with $L=5$ time steps,

$$
\begin{aligned}
& U=W V X Y Z=\left(W_{12} \otimes W_{3} \otimes W_{4} \otimes W_{5} \otimes W_{6}\right) \\
& \quad \ldots\left(Z_{1} \otimes Z_{2} \otimes Z_{3} \otimes Z_{4} \otimes Z_{56}\right) .
\end{aligned}
$$

In the case of six qubit system $(n=2-$ see Fig. $4 a)$ some deviations from the CUE results are visible, while in the case of six qutrits $(n=3$, Fig. $4 \mathrm{~b})$ the distribution follows predictions of random matrices with a good accuracy. Hence Wigner-like spectral properties of the 
evolution operator can be obtained under very weak assumptions on the interaction, as the first and the last subsystems are coupled only indirectly by the $L$-step interaction.

\section{Eigenvectors of graph-structured unitary matrices}

Matrices of eigenvectors of random unitary matrices are known to be distributed according to the Haar measure on the unitary group [2]. It is interesting to analyze statistical properties of eigenvectors of unitary matrices associated with a given graph and compare them with predictions for random matrices.

Let us write the eigenequation of a unitary matrix as $U\left|\chi_{j}\right\rangle=\mathrm{e}^{\mathrm{i} \vartheta_{j}}\left|\chi_{j}\right\rangle$. The eigenstates are normalized $\left\langle\chi_{j} \mid \chi_{j}\right\rangle=1$ for $j=1, \ldots, N$, so the complex expansion coefficients $\chi_{j i}$ of the state $\left|\chi_{j}\right\rangle$ in the computational basis satisfy $\sum_{i=1}^{N}\left|\chi_{j i}\right|^{2}=1$ for $j=1, \ldots, N$. These $N$ non-negative numbers form thus a probability vector, the distribution of which can be characterized by its Shannon entropy.

\subsection{Eigenvector entropy}

For any unitary matrix $U$ one defines the eigenvector entropy as the average Shannon entropy of a single eigenvector:

$$
H_{\mathrm{ev}}(U) \equiv-\frac{1}{N} \sum_{i, j}\left|\chi_{j i}\right|^{2} \log \left|\chi_{j i}\right|^{2} .
$$

Let us note that this quantity coincides with the entropy of the unistochastic matrix [16] corresponding to the unitary matrix of eigenvectors of $U$. The mean entropy of eigenvector or a random unitary matrix of order $N$, coincides with the mean entropy of a random complex vector $[17,18]$ :

$$
\left\langle H_{\mathrm{el}}\right\rangle=\psi(N+1)-\psi(2)=\sum_{j=2}^{N} \frac{1}{j} .
$$

Here $\psi(x)$ denotes the digamma function, $\mathrm{d} \ln \Gamma(x) / \mathrm{d} x$.

A comparison of the distribution of eigenvector entropy for graph-structured and unstructured random unitary matrices is presented in Fig. 5. Our numerical investigations show that statistical properties of eigenvectors of unitary matrices associated to connected graphs coincide for large dimensions with the prediction of random CUE matrices.

\subsection{Entropy and purity of a reduced state}

A unitary matrix $U$ associated to a graph acts on the Hilbert space with a tensor product structure and corresponds to composed systems. Let us divide the system into two parts, labeled by $A$ and $B$. The eigenvectors $|\chi\rangle$ of $U$ can be considered as pure states of a bi-partite system $A B$. One can thus investigate their entanglement entropy with respect to the partition $A-B$, equal to the von Neumann entropy $H(\sigma)=-\operatorname{Tr} \sigma \ln \sigma$ of the reduced state, $\sigma_{A}=\operatorname{Tr}_{B}|\chi\rangle\langle\chi|$.

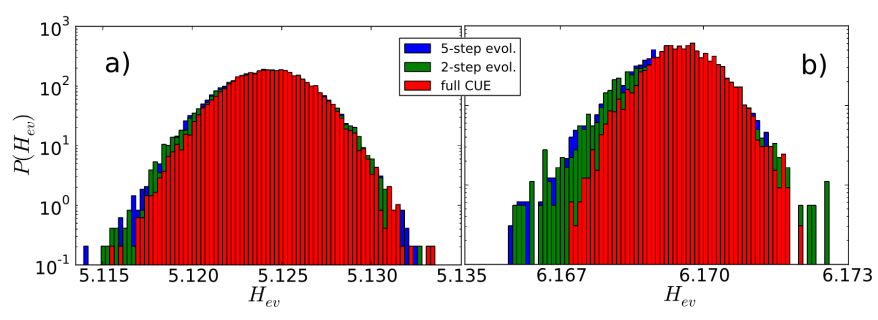

Fig. 5. Distribution of eigenvector entropy $P\left(H_{\mathrm{ev}}(U)\right)$ for unitary matrices $U$ associated to (a) a chain consisting of 4 subsystems, $U=W V=\left(W_{12} \otimes W_{34}\right)\left(V_{1} \otimes V_{23} \otimes\right.$ $\left.V_{4}\right)$ realized for $n=4$, sample size 20000 (green) and the corresponding three-step evolution (blue) and (b) six-particle chain with a two-step local interaction given by Eq. (8) obtained for $n=3$, sample size 50000 (green), and its five-step generalization (blue). The larger dimension $n$ of a single system, the better agreement with the CUE data (red) obtained for the same total dimension $N$.

For random vectors of the size $N_{A} N_{B}$ the average entropy of a subsystem of dimension $N_{A}$ reads [19, 20]:

$$
\langle H\rangle \approx \log N_{A}-\frac{N_{A}-1}{2 N_{B}} .
$$

Here it is assumed that the dimension $N_{A}$ of the reduced state is large and $N_{B} \geq N_{A}$ so that the maximal entropy $H_{\text {max }}$ is equal to $\log N_{A}$. If both subsystems are equal $N_{B}=N_{A}=\sqrt{N}$, the reduced states $\sigma_{A}$ are distributed uniformly according to the Hilbert-Schmidt measure in the set of mixed quantum states and the average entropy is $\langle H\rangle_{\mathrm{HS}} \approx \frac{1}{2} \log N-\frac{1}{2}$. By definition it is equal to the average entanglement entropy of random pure states of size $N=N_{A}^{2}$.

In order to provide an alternative characterization of degree of mixing of a quantum state one often uses $p u-$ rity, $\operatorname{Tr} \sigma^{2}$, equal to unity for a pure state. This quantity applied to a reduced state $\sigma_{A}=\operatorname{Tr}_{B}|\chi\rangle\langle\chi|$, carries information about entanglement of a bi-partite state $|\chi\rangle$. For pure random states of size $N=N_{A} N_{B}$ the average purity is $[21,18]$ :

$$
\langle R\rangle=\frac{N_{A}+N_{B}}{N_{A} N_{B}+1} .
$$

Average entanglement entropy of eigenvectors of random unitary matrices associated to exemplary graphs are shown in Fig. 6 and Fig. 7 while the average purity is presented in Fig. 8b. In the case of connected graphs the entropy of entanglement and purity of the reduced eigenvectors of associated unitary matrices coincide thus with properties of random vectors described by random matrices.

\subsection{Projection to a smaller subspace}

As eigenvectors $|\chi\rangle$ of $U$ denote pure states of a multipartite systems, it is of interest to investigate properties of their projections onto a smaller subspace. We have performed such a procedure for a system of three particles (see Fig. 2c), with unequal dimensions of the subspaces, $N_{A}=N_{C} \neq N_{B}$. After choosing one of the basis 


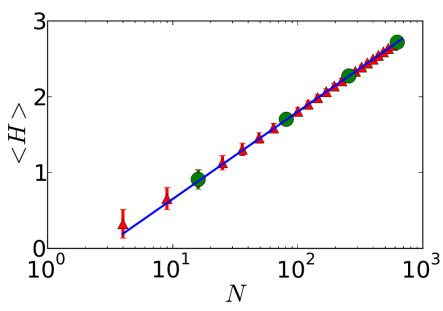

Fig. 6. Mean entanglement entropy $\langle H\rangle$ of the eigenvectors of a structured unitary matrix $U$, versus its size $N$. The red triangles denote the system of two particles, Fig. 2b, and the green circles - four particles, Fig. 2d. In all the cases the dimensions of the two subsystems $A$ and $B$ are equal, $N_{A}=N_{B}=\sqrt{N}$. The solid line follows from the prediction for CUE matrices (Eq. (11), with $N_{A}=N_{B}=\sqrt{N}$ ).

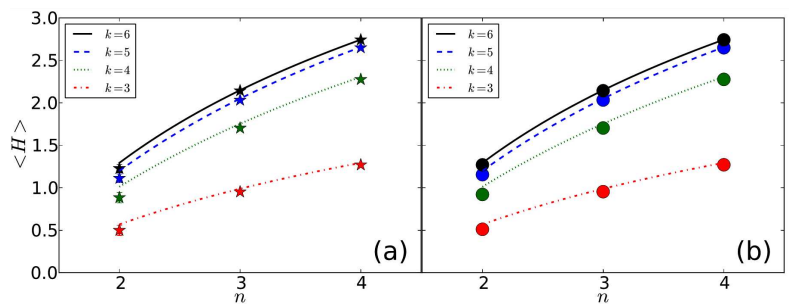

Fig. 7. Linear chains of subsystems of length $k=$ $3,4,5,6$. (a) Average entropy $\langle H\rangle$ of the subsystem consisting of two peripheral particles, versus the dimension $n$ of the Hilbert space of a single particle (symbols) and predictions of random matrices following from (11) lines. (b) Comparison with the non-structured random unitary matrices of order $N=n^{k}$.

vectors $\left|i_{B}\right\rangle$ of $\mathcal{H}_{B}$, the eigenstates $\left|\Psi_{A B C}\right\rangle=|\chi\rangle$ were projected onto the subspace $\mathcal{H}_{A} \otimes \mathcal{H}_{C}$,

$$
\left|\tilde{\Psi}_{A C}\right\rangle \equiv\left\langle i_{B} \mid \Psi_{A B C}\right\rangle \text {. }
$$

Average entanglement between particles $A$ and $C$ was characterized by the purity and the von-Neumann entropy of the reduced state $\sigma_{A}=\operatorname{Tr}_{C}\left|\tilde{\Psi}_{A C}\right\rangle\left\langle\tilde{\Psi}_{A C}\right|$. The averaging is performed over all $N_{B}$ basis vectors in the Hilbert space $\mathcal{H}_{B}$ as well as over several realizations of

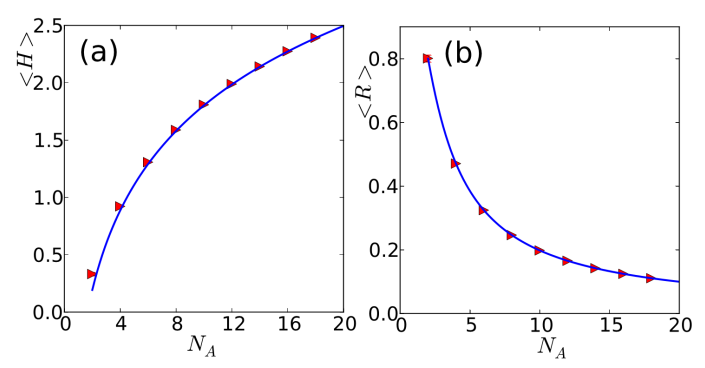

Fig. 8. Properties of eigenvectors of matrices associated to a three-chain projected onto a single subspace as in Eq. (12) as a function of its dimension $n=N_{A}$ : (a) mean entropy $\langle H\rangle$ and (b) mean purity $\langle R\rangle$ compared with predictions of random matrices. the corresponding random matrix.

Interestingly, entanglement between the peripheral particles does not depend on the dimension of the central particle, which acts as a proxy of interactions. As shown in Fig. 8, entropy and purity of the projected eigenvectors exhibit the CUE-like behavior.

\section{Distribution of matrix elements}

Although unitary matrices related to connected graphs display statistical properties of spectra and eigenvectors according to predictions of random matrices, the distribution of their elements is different. To describe quantitatively the distribution of entries of $U$ we use the element entropy,

$$
H_{\mathrm{el}}(U) \equiv-\frac{1}{N} \sum_{i j}\left|u_{i j}\right|^{2} \log \left|u_{i j}\right|^{2} .
$$

For any unitary matrix with a tensor product structure the element entropy is additive,

$$
H_{\mathrm{el}}(U \otimes V)=H_{\mathrm{el}}(U)+H_{\mathrm{el}}(V) .
$$

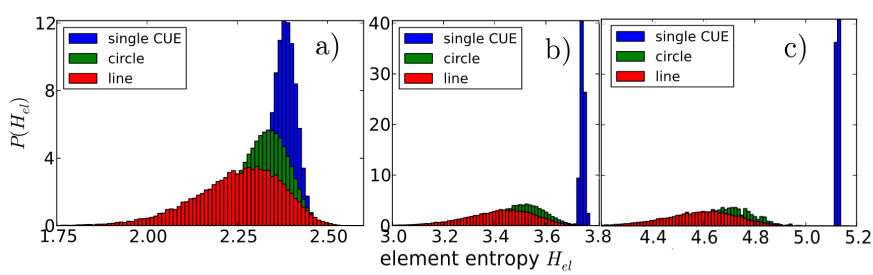

Fig. 9. Distribution of the element entropy $H_{\mathrm{el}}$ for random matrices associated to a chain (such as in Fig. 2c; red histogram), circle (such as in Fig. 2d or e; green) and random CUE matrices (blue). Dimension of a single subsystem is $n=2$, while the number of particles is (a) $k=4$, (b) $k=6$, (c) $k=8$.

The distribution of the element entropy for random matrices associated to exemplary connected graphs is shown in Fig. 9. Any construction of a CUE matrix of order $N$ requires more independent random numbers than to generate smaller matrices necessary to construct matrices associated to a graph. Therefore the distribution of the element entropy is narrower for CUE matrices than for matrices associated to a graph and it allows one to distinguish between structured and unstructured random matrices.

\section{Concluding remarks}

We proposed a construction of random unitary matrices which, interpreted as representations of the Hamiltonian evolution operators, correspond to various scenarios of the interaction between quantum objects. The structure of such matrices can be determined by a graph in two different ways. In the first method each bond represents two interacting subsystems, while the other phase of the interaction couples all subsystems connected by a single 
vertex. In the alternative method the vertices represent individual subsystems and the edges represent interaction between them. In this work we focus on the case, in which no details concerning the interaction Hamiltonian are known, so the interaction is modeled by random unitary matrices generated according to the Haar measure on the unitary group.

Our numerical results support the conjecture that the spectral properties of structured unitary matrices associated to a connected graph are well described by predictions of CUE random matrices. This concerns the level density $P(\theta)$, the nearest neighbour spacing distribution $P(S)$, and also statistical properties of eigenvectors. On the other hand, the simplest method do distinguish between ensembles of structured unitary matrices and CUE is to analyze statistics of their elements and compare e.g. the element entropy.

Statistical properties of elements of random matrices associated to a graph $\Gamma$ are determined by its topology. Analyzing statistics of the elements and the traces of unitary matrices associated to two different graphs in several cases one can distinguish between these two graphs. However, in general it seems not to be possible to determine in this way, whether two investigated graphs are isomorphic.

Making use of unitary random matrix associated to a graph $\Gamma$ one can act with it on a given separable pure state and arrive at a random pure state, $|\Psi\rangle=U \mid 1 \otimes$ $\ldots \otimes 1\rangle$. In this way one obtains an ensemble of random states associated to a graph $\Gamma$, which can be considered as a generalization of the ensembles investigated in $[3,4]$. On the other hand, the above construction of an ensemble of quantum states corresponding to a graph differs from the notion of quantum graphs studied by Gnutzmann and Smilansky [22] or these related to microwave experiments investigated in [23, 24].

As the standard model discussed in this work corresponds to a two-step interaction, represented by a two-colour graph, it can be generalized for an arbitrary number of $L$ time steps, and the corresponding $L$-colour graphs. It is worth to emphasize that even in the case of the chain graphs, with local interaction coupling two neighbouring sites only, CUE-like spectral properties are observed for large system size provided the number of the time steps $L$ is sufficiently large. This means that the randomness can be transferred by a stepwise nearest neighbour interaction, from the first subsystem to the last one.

Random unitary matrices play an important role in various protocols of quantum information processing. However, the number of quantum gates necessary to implement a random unitary matrix grows exponentially with the number of qubits [25]. As a substitute one may construct various pseudorandom matrices, the statistical properties of which should be similar to these of CUE [25-27].

The scheme of random unitary matrices associated with a graph, developed in this work, can be thus directly applied to construct large random unitary matrices out of a few much smaller unitary matrices of order. Consider for instance random matrices

$$
\begin{aligned}
& U=V W=\left(V_{2,3} \otimes V_{4,5} \otimes \cdots \otimes V_{k, 1}\right)\left(W_{1,2} \otimes W_{3,4}\right. \\
& \left.\quad \otimes \cdots \otimes W_{k-1, k}\right)
\end{aligned}
$$

associated with a two colour ring of $k-1$ subsystems interacting with one neighbour in phase one (bonds of a first colour) and with the other on phase two (bonds of the second colour). This natural extension of a six-partite system shown in Fig. 2e allows one to obtain random unitary matrices of size $N=n^{k}$ out of $k$ smaller CUE matrices of size $n^{2}$. As discussed in Appendix the generation time of such graph-structured matrices can be shorter compared to the standard CUE sampling.

\section{Acknowledgments}

It is a pleasure to thank Benoit Collins, Marek Kuś, Ion Nechita, and Tomasz Tkocz for fruitful discussions and helpful remarks. Financial support by the Polish National Science Centre under the contract number DEC-2011/01/M/ST2/00379 and by the Deutsche ForschungsGemeinschaft under the project SFB Transregio-12 is gratefully acknowledged.

\section{Appendix: Time costs of the matrix generation}

The calculations were performed on two PCs, with processors Intel(R) Core(TM)2 CPU T5500 @ $1.66 \mathrm{GHz}$ and Intel(R) Xeon(R) CPU X3430@ 2.40 GHz. The computer program was written in $\mathrm{C}++$ and used the Armadillo [28] linear algebra library. The sampling of CUE was implemented according to the algorithm proposed by Mezzadri [11], based on the QR decomposition. In a single run, $T$ matrices were generated and then diagonalized to obtain the eigenvalues.

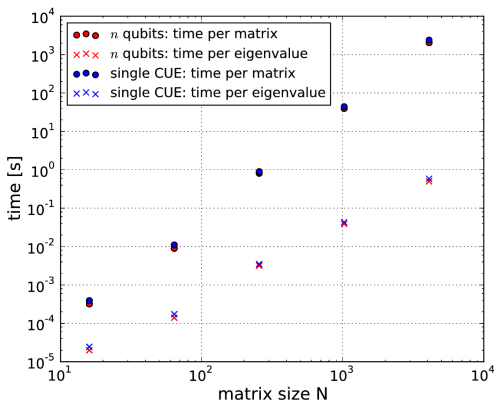

Fig. 10. Time costs of the matrix generation: line-structured system of $n$ qubits, see Fig. $2 \mathrm{~b}$ (red points) vs. direct construction of random CUE matrices (blue points).

As shown in Fig. 10 and Table, construction of random matrices associated to a graph costs less time than the corresponding CUE matrix obtained by the Mezzadri algorithm. However, to investigate spectral properties of a matrix obtained one needs to diagonalise it, and for 
TABLE

Comparison of the time required to generate random matrices associated to a square graph and obtained by a CUE algorithm. Processor: Intel(R) Xeon(R) CPU X3430 @ $2.40 \mathrm{GHz}$.

\begin{tabular}{|c|c|c|c|}
\hline Matrix type & \# matrices & Time [s] & Rel. time $[\%]$ \\
\hline $\begin{array}{l}\text { CUE, } N=256 \\
\text { square graph, } \\
4 \text { matrices } \\
\text { of size } n=4\end{array}$ & 1000 & $\begin{array}{l}85.93 \\
47.24\end{array}$ & 100 \\
\hline $\begin{array}{l}\text { CUE, } N=625 \\
\text { square graph, } \\
4 \text { matrices } \\
\text { of size } n=5\end{array}$ & $\begin{array}{l}100 \\
100\end{array}$ & $\begin{array}{l}100.27 \\
62.26\end{array}$ & $\begin{array}{l}100 \\
62.1\end{array}$ \\
\hline
\end{tabular}

a larger matrix size $N$ the computing time needed to construct a random matrix is dominated by the diagonalization time.

\section{References}

[1] F. Haake, Quantum Signatures of Chaos, 3rd ed., Springer, Berlin 2006.

[2] M.L. Mehta, Random Matrices, Academic Press, Amsterdam 2004.

[3] B. Collins, I. Nechita, K. Życzkowski, J. Phys. A 43, 275303 (2010).

[4] B. Collins, I. Nechita, K. Życzkowski, J. Phys. A 46, 305302 (2013).

[5] F. Verstraete, J.I. Cirac, Phys. Rev. A 70, 060302 (2004).

[6] F. Verstraete, M.M. Wolf, D. Perez-Garcia, J.I. Cirac, Phys. Rev. Lett. 96, 220601 (2006).

[7] S. Perseguers, J.I. Cirac, A. Acin, M. Lewenstein, J. Wehr, Phys. Rev. A 77, 022308 (2008).

[8] S. Perseguers, D. Cavalcanti, G.J. Lapeyre, Jr., M. Lewenstein, A. Acin, Phys. Rev. A 81, 032327 (2010).

[9] P. Pakoński, G. Tanner, K. Życzkowski, J. Stat. Phys. 111, 1331 (2003).
[10] K. Życzkowski, M. Kuś, J. Phys. A 27, 4235 (1994).

[11] F. Mezzadri, Notic. AMS 54, 592 (2007).

[12] T. Tkocz, M. Smaczyński, M. Kuś, O. Zeitouni, K. Zyczkowski, Random Matric. Theory Appl. 1, 1250009 (2012).

[13] T. Tkocz, Electron. Commun. Probab. 18, 1 (2013).

[14] M. Smaczyński, T. Tkocz, M. Kuś, K. Życzkowski, Phys. Rev. E 88, 052902 (2013).

[15] M. Poźniak, K. Życzkowski, M. Kuś, J. Phys. A Math. Gen. 31, 1059 (1998).

[16] K. Życzkowski, M. Kuś, W. Słomczyński, H. Sommers, J. Phys. A 36, 3425 (2003).

[17] K. Jones, J. Phys. A 23, L1247 (1990).

[18] I. Bengtsson, K. Życzkowski, Geometry of Quantum States: An Introduction to Quantum Entanglement, Cambridge Univ. Press, Cambridge 2006.

[19] D.N. Page, Phys. Rev. Lett. 71, 1291 (1993).

[20] K. Życzkowski, H. Sommers, J. Phys. A 34, 7111 (2001).

[21] E. Lubkin, J. Math. Phys. 19, 1028 (1978).

[22] S. Gnutzmann, U. Smilansky, Adv. Phys. 55, 527 (2006).

[23] O. Hul, S. Bauch, P. Pakoński, N. Savytskyy, K. Życzkowski, L. Sirko, Phys. Rev. E 69, 056205 (2004).

[24] M. Ławniczak, A. Borkowska, O. Hul, S. Bauch, L. Sirko, Acta Phys. Pol. A 120, A185 (2011).

[25] J. Emerson, Y. Weinstein, M. Saraceno, S. Lloyd, D. Cory, Science 302, 2098 (2003).

[26] M. Žnidarič, Phys. Rev. A 76, 012318 (2007).

[27] Y.S. Weinstein, "Pseudo-Random Circuits from Clifford Plus T-Gates," arXiv:1309.3943, 2013.

[28] C. Sanderson, "Armadillo: An Open Source C++ Linear Algebra Library for Fast Prototyping and Computationally Intensive Experiments," tech. rep., NICTA, 2010 . 